\title{
Flambage et vibrations non-linéaires d'une plaque stratifiée piézoélectrique. Application à un capteur de masse MEMS
}

\author{
Olivier Thomas ${ }^{1, a}$, Liviu Nicu ${ }^{2}$ et Cyril TouzÉ ${ }^{3}$ \\ 1 Laboratoire de Mécanique des Structures et des Systèmes Couplés, EA 3196, Cnam, 2 rue Conté, 75003 Paris, France \\ 2 Laboratoire d'Analyse et Architecture des Systèmes, UPR 8001, 7 Av. du Colonel Roche, 31077 Toulouse, France \\ 3 Unité de Mécanique, ENSTA, Ch. de la Hunière, 91761 Palaiseau Cedex, France
}

Reçu le 20 avril 2009

\begin{abstract}
Résumé - L'objet de ce travail est de présenter les premiers résultats de modélisation et simulation du comportement vibratoire non-linéaire d'une plaque circulaire stratifiée munie de couches en matériau piézoélectrique, élément principal d'un capteur de masse MEMS. La modélisation permet de prendre en compte (i) le couplage électromécanique lié à la pastille piézoélectrique, (ii) la structure stratifiée des membranes, (iii) l'état précontraint au repos de la membrane et le flambage éventuel et (iv) les vibrations non-linéaires qui produisent un effet raidissant ou assouplissant. Le modèle non-linéaire et sa résolution, par méthode de continuation et équilibrage harmonique, sont présentés, pour dégager le comportement de la membrane en régime forcé.
\end{abstract}

Mots clés : Plaque stratifiée / non-linearités géométriques / flambage / vibrations / piézoélectrique

\begin{abstract}
Buckling and non-linear vibrations of a piezoelectric stratified plate. Application to a MEMS mass sensor. This study proposes a model of a circular, non-symetrically laminated, piezoelectric plate, subjected to non-linear large amplitude vibrations. This model is built to simulate the behavior of a MEMS bio-sensor, designed to detect, automatically and autonomously, the presence of a given molecule in an aqueous solution. Because of both the laminated structure and the fabrication process, prestresses are observed, with different signs and intensities from one layer to another. This is responsible of a non-planar deformed geometry of the plate at rest. Moreover, experimental results show that a geometrically non-linear response is observed, with curved freqeuncy responses. A continuous nonlinear model of the von-Kármán type is used and discretized by an expansion of the solution onto the mode shape basis of the plate without prestresses. It's response is numerically computed by a continuation method, in order to predict the system's non-linear behavior.
\end{abstract}

Key words: Stratified plate / geametrical non-linearities / buckling / vibrations / piezoelectric

\section{Introduction}

Dans ce travail, on se propose d'étudier le comportement vibratoire non-linéaire d'une plaque circulaire stratifiée en matériau piézoélectrique. Il s'agit de modéliser et de simuler le fonctionnement d'un biocapteur MEMS (Micro-Electro Mechanical System) permettant de détecter, de façon automatique et autonome, la présence d'une molécule donnée contenue dans une solution aqueuse.

Le bio-capteur qu'il s'agit d'étudier est constitué de plusieurs membranes circulaires, d'environ $200 \mu \mathrm{m}$ de

\footnotetext{
${ }^{a}$ Auteur pour correspondance : olivier.thomas@cnam.fr
}

diamètre et d'épaisseur de l'ordre du $\mu \mathrm{m}$, inscrites dans une plate-forme de silicium (Fig. 1). Ces membranes sont entraînées en vibration sur leur mode fondamental grâce à l'action d'une pastille piézoélectrique. Elles sont de plus recouvertes d'une espèce biologique (le ligand) capable de reconnaître et d'immobiliser une molécule donnée (la biomolécule). Lorsque les membranes sont en contact avec une solution aqueuse contenant des biomolécules, celles-ci sont captées par le ligand et les membranes s'en trouvent alors alourdies. En détectant les vibrations des membranes, on peut mesurer la variation de fréquence de résonance qui s'ensuit et ainsi estimer la quantité de biomolécule présente dans le liquide. Il est possible 


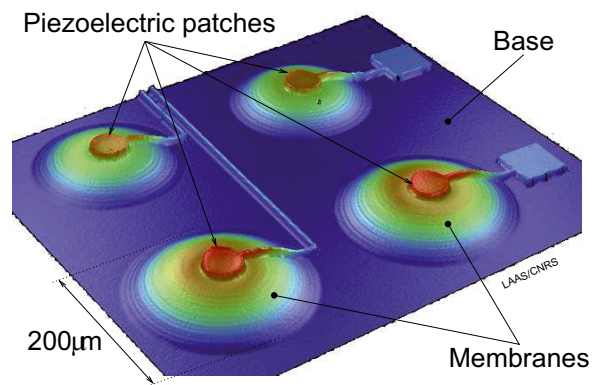

Fig. 1. Un bio-capteur constitué d'une matrice $2 \times 2$ de membranes résonantes.

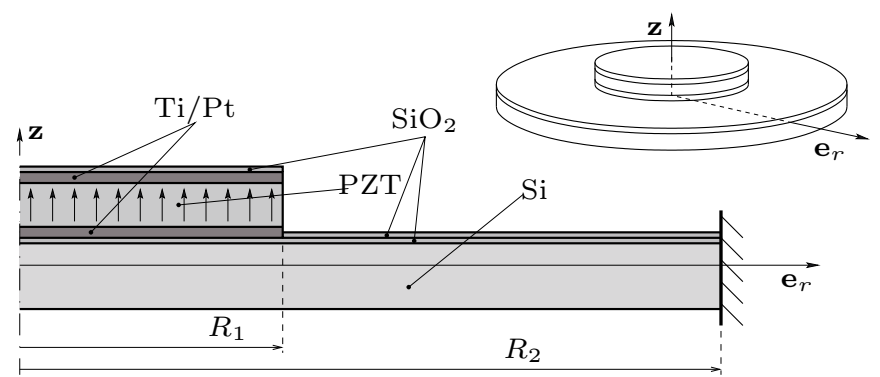

Fig. 2. Section droite d'une membrane stratifiée.

d'utiliser d'autres géométries de capteur, notamment en forme de poutre encastrée/libre [1]. Néanmoins, le choix de membranes circulaires paraît être prometteur, notamment en terme de facteur de qualité, et c'est ce qui motive la présente étude [2].

À l'heure actuelle, alors que les technologies pour fabriquer un tel système sont bien maîtrisées [3], son comportement mécanique est encore mal connu et une modélisation mécanique complète est nécessaire pour affiner sa mise au point [2]. La première difficulté vient de la structure stratifiée des membranes (Fig. 2). En effet, du fait du procédé de fabrication, des précontraintes positives et négatives, différentes d'une couche à l'autre, sont présentes à l'état de repos. La seconde difficulté provient de la pastille piézoélectrique circulaire placée au milieu de la membrane, qui est polarisée après insertion dans le système, produisant ainsi des précontraintes additionnelles. La première conséquence de ces diverses précontraintes est que l'état de repos de la membrane n'est pas plan (Fig. 3). C'est cette position flambée qu'il s'agit de décrire en premier lieu.

Ensuite, le fonctionnement de la membrane consiste à l'exciter en vibration sur son mode fondamental, grâce à la pastille piézoélectrique, pour mesurer une variation de fréquence de résonance produite par un alourdissement de la membrane, dû à la capture de molécules à doser. Le modèle que l'on cherche à établir doit donc permettre d'évaluer les fréquences de résonance de la structure autour de la position de repos flambée.

Enfin, pour obtenir une mesure de variation de fréquence de résonance avec un rapport signal sur bruit correct, des premières mesures ont montré qu'il était

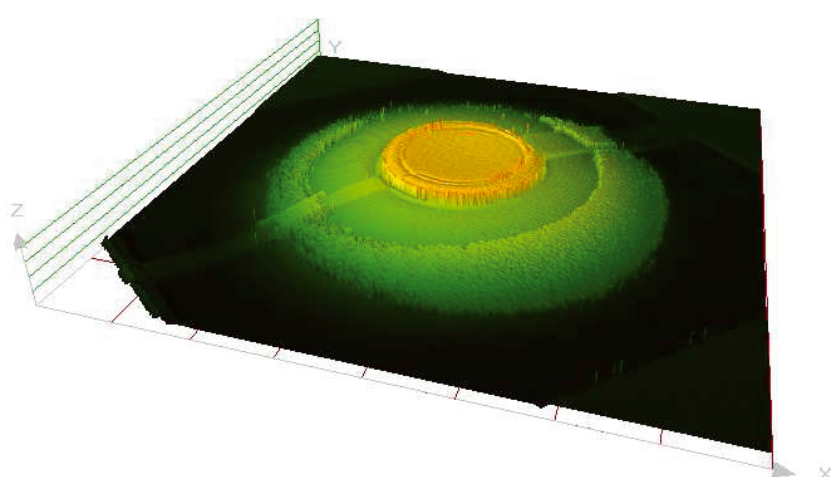

Fig. 3. Géométrie flambée de la membrane au repos (résultats expérimentaux).

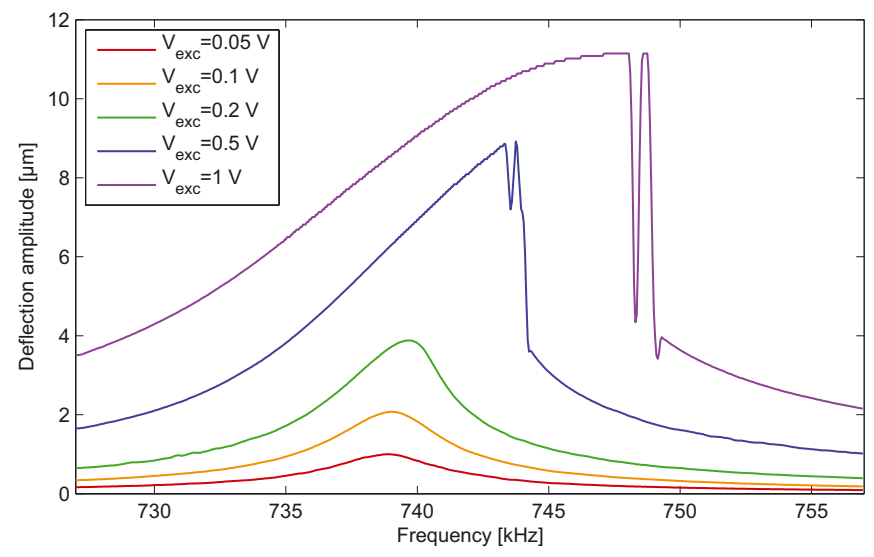

Fig. 4. Réponse dynamique vibratoire non-linéaire (résultats expérimentaux).

nécessaire de faire fonctionner la membrane en régime de vibration non-linéaire de grande amplitude. Notamment, des courbes de résonance incurvées ont été observées (Fig. 4), montrant des effets raidissant (courbes incurvées vers les hautes fréquences) et assouplissant (courbe incurvée vers les basses fréquences) non-négligeables.

Dans cette étude, on se propose de mettre en place le cadre général de modélisation. Ensuite, des résultats de simulations sont proposés, dans le cas d'une structure stratifiée symétrique dans l'épaisseur. La position flambée des membranes, sous l'action d'une précontrainte, est simulée, ainsi que la variation correspondante des fréquences propres et des déformées modales. Ensuite, le comportement en vibrations non-linéaires autour de la position d'équilibre flambée est étudié, pour plusieurs valeurs de la précontrainte. On montre en particulier que le comportement des membranes est raidissant lorsque la membrane n'est pas flambée, alors qu'il devient assouplissant lorsque la membrane flambe.

\section{2 Équations du mouvement}

On considère une plaque circulaire stratifiée munie en son centre d'une pastille circulaire piézoélectrique 
(Fig. 2). Les couches de silicium ( $\mathrm{Si}$ ), oxyde de silicium $\left(\mathrm{SiO}_{2}\right)$ et les électrodes de titane/platine (Ti/Pt) sont homogènes et isotropes. Ces deux électrodes, branchées à un générateur extérieur, permettent de créer dans la couche de matériau piézoélectrique (PZT) un champ électrique dirigé suivant $z$. Cela permet d'une part de polariser la couche PZT et d'autre part de la déformer dans la direction longitudinale (suivant $e_{r}$ ) pour entraîner le système en vibration. La couche PZT est constituée d'un matériau homogène et isotrope transverse dans le plan normal à $z$. Pour la partie mécanique, on utilise le modèle classique des plaques stratifiées [4] avec des déformations de type von Kármán. Pour la partie électrique, on suppose que le champ électrique est constant suivant $z$. Tout point du plan moyen de la plaque est repéré par ses coordonnées polaires $(r, \theta)$. La géométrie du système est axisymétrique et composée de deux zones : la zone circulaire centrale $\mathcal{D}_{1}=\left[0, R_{1}\right] \times[0,2 \pi[$ et la zone annulaire périphérique $\mathcal{D}_{2}=\left[R_{1}, R_{2}\right] \times[0,2 \pi[$. Le domaine de la plaque est noté $\mathcal{D}=\mathcal{D}_{1} \cup \mathcal{D}_{2}$. La plaque est supposée encastrée sur son pourtour, en $r=R_{2}$.

En supposant que la transformation que subit la plaque est axisymétrique, les déformations en un point de coordonnées $(r, \theta, z)$ s'écrivent $\varepsilon=\left\{\varepsilon_{r} \varepsilon_{\theta}\right\}^{\mathrm{T}}=\boldsymbol{\epsilon}+z \boldsymbol{\kappa}$, avec $\boldsymbol{\epsilon}=\left\{\epsilon_{r} \epsilon_{\theta}\right\}^{\mathrm{T}}$ et $\boldsymbol{\kappa}=\left\{\kappa_{r} \kappa_{\theta}\right\}^{\mathrm{T}}$ et

$$
\begin{aligned}
& \epsilon_{r}=u_{r, r}+1 / 2 w_{, r}^{2}, \\
& \epsilon_{\theta}=u_{r} / r, \quad \kappa_{r}=-w_{, r r}, \quad \kappa_{\theta}=-w_{, r} / r
\end{aligned}
$$

où $u_{r}$ est le déplacement radial et $w$ le déplacement transverse d'un point du plan moyen. ${ }^{\circ}, r$ représente la dérivée de o par rapport à $r$. La loi de comportement s'écrit :

$$
\boldsymbol{\sigma}=\boldsymbol{Q}(\boldsymbol{\epsilon}+z \boldsymbol{\kappa})-\sigma_{0 P} \mathbf{1}, \text { avec } \sigma_{0 P}=\sigma_{0}+\frac{e_{31}}{h_{p}} V(t)
$$

où $\boldsymbol{\sigma}=\left\{\sigma_{r} \sigma_{\theta}\right\}^{\mathrm{T}}$ est le vecteur des contraintes, $\boldsymbol{Q}$ est l'opérateur de Hooke et $\mathbf{1}=\{11\}^{\mathrm{T}}$. $\sigma_{0}$ représente les précontraintes de fabrications, $V(t)$ est la différence de potentiel électrique entre les électrodes, $e_{31}$ est la constante piézoélectrique et $h_{p}$ est l'épaisseur de la couche PZT. En intégrant l'équation (2) suivant l'épaisseur, on obtient la loi de comportement, en terme des efforts de membrane $\boldsymbol{N}=\left\{N_{r} N_{\theta}\right\}^{\mathrm{T}}$ et des moments fléchissants $\boldsymbol{M}=\left\{M_{r} M_{\theta}\right\}^{\mathrm{T}}$ et des matrices $\boldsymbol{A}, \boldsymbol{B}$ et $\boldsymbol{D}[4]$ :

$$
\left(\begin{array}{l}
\boldsymbol{N} \\
\boldsymbol{M}
\end{array}\right)=\left(\begin{array}{ll}
\boldsymbol{A} & \boldsymbol{B} \\
\boldsymbol{B} & \boldsymbol{D}
\end{array}\right)\left(\begin{array}{l}
\boldsymbol{\epsilon} \\
\boldsymbol{\kappa}
\end{array}\right)-\left(\begin{array}{l}
N_{0 P} \mathbf{1} \\
M_{0 P} \mathbf{1}
\end{array}\right)
$$

où $N_{0 P}$ et $M_{0 P}$ sont créés par les précontraintes et les efforts piézoélectriques issus de $\sigma_{0 P}$. En inversant l'équation (3), on obtient [5] :

$$
\left(\begin{array}{c}
\boldsymbol{\epsilon} \\
\boldsymbol{M}
\end{array}\right)=\left(\begin{array}{cc}
\boldsymbol{A}^{*} & \boldsymbol{B}^{*} \\
-\boldsymbol{B}^{* \mathrm{~T}} & \boldsymbol{D}^{*}
\end{array}\right)\left(\begin{array}{c}
\boldsymbol{N} \\
\boldsymbol{\kappa}
\end{array}\right)+\left(\begin{array}{c}
A^{+} N_{0 P} \mathbf{1} \\
-\tilde{M}_{0 P} \mathbf{1}
\end{array}\right)
$$

avec $\boldsymbol{A}^{*}=\boldsymbol{A}^{-1}, \boldsymbol{B}^{*}=-\boldsymbol{A}^{-1} \boldsymbol{B}, \boldsymbol{D}=\boldsymbol{D}+\boldsymbol{B} \boldsymbol{B}^{*}, A^{+}=$ $A_{11}^{*}+A_{12}^{*}, \tilde{M}_{0 P}=B^{+} N_{0 P}+M_{0 P}$ et $B^{+}=B_{11}^{*}+B_{12}^{*}$.
En injectant les équations (4) dans l'équation d'équilibre transverse et la condition de compatibilité, après adimensionnement et quelques calculs, on obtient :

$$
\left\{\begin{array}{l}
L_{D}(w)+\varepsilon L_{B}(F)+\Delta M_{0 P}+\varepsilon \Delta\left(B^{+} N_{0 P}\right)+\bar{m} \ddot{w}= \\
\varepsilon L(w, F)+p \\
L_{A}(F)-L_{B}(w)+\Delta\left(A^{+} N_{0 P}\right) l=-1 / 2 L(w, w)
\end{array}\right.
$$

où $L_{A}, L_{B}$ et $L_{D}$ sont des opérateurs différentiels qui tiennent compte de la discontinuité des matrices $\boldsymbol{A}$, $\boldsymbol{B}$ et $\boldsymbol{D}$ en $r=R_{1}$ et $\varepsilon$ un paramètre adimensionné. Lorsque ces dernières sont indépendantes de $r$, $L_{A}(\circ)=A_{11}^{*} \Delta \Delta \circ, L_{B}(\circ)=B_{12}^{*} \Delta \Delta \circ$ et $L_{D}(\circ)=$ $D_{11}^{*} \Delta \Delta \circ$ avec $\Delta$ l'opérateur laplacien. $L(\circ, \circ)$ est l'opérateur bilinéaire classique associé aux équations de von Kármán [6]. $\bar{m}$ est la masse surfacique de la plaque, $\ddot{w}$ est la dérivée seconde de $w$ par rapport au temps et $p$ une pression extérieure. $F$ est une fonction d'Airy, relié à $\boldsymbol{N} \operatorname{par} N_{r}=F_{, r} / r$ et $N_{\theta}=F_{, r r}$.

Les conditions aux limites imposent d'une part que $w$ et $F$ soient finis en $r=0$ et d'autre part que sur le bord, en $r=1: w=0, w_{, r}=0, \epsilon_{\theta}=0$ et $\epsilon_{r}-\left(r \epsilon_{\theta}\right)_{r}=0$.

\section{Développement modal}

De la même manière que dans [6], on cherche le déplacement $w$ et la fonction d'Airy $F$ sous la forme des développements sur les modes propres de la structure :

$$
\begin{aligned}
w(r, t) & =\sum_{i=1}^{N_{w}} \Phi_{i}(r) q_{i}(t) \\
F(r, t) & =\sum_{i=1}^{N_{w}} \Psi_{j}(r) q_{i}(t)+\sum_{j=1}^{N_{F}} \Upsilon_{j}(r) \eta_{j}(t)
\end{aligned}
$$

où $N_{w}$ est le nombre de modes transverses et $N_{F}$ le nombre de modes de membrane. Les $\left(\Phi_{i}, \Psi_{i}, \omega_{i}\right)$ sont précisément les modes propres de la structure en courtcircuit $(V \equiv 0)$ et non précontrainte $\left(N_{0 P}=M_{0 P} \equiv 0\right)$. Ils sont solutions de :

$$
\left\{\begin{array}{r}
L_{D}\left(\Phi_{s}\right)+\varepsilon L_{B}\left(\Psi_{s}\right)-\bar{m} \omega_{s}^{2} \Phi_{s}=0 \\
L_{A}\left(\Psi_{s}\right)-L_{B}\left(\Phi_{s}\right)=0
\end{array}\right.
$$

associées de conditions aux limites encastrées. Les $\left(\Upsilon_{j}, \zeta_{j}\right)$ sont solutions de :

$$
L_{A}\left(\Psi_{s}\right)-\zeta_{s}^{4} \Upsilon_{s}=0
$$

associées de conditions aux limites appropriées.

En introduisant les équations (6) dans les équations du mouvement (5a) et $(5 \mathrm{~b})$ et en utilisant les propriétés d'orthogonalité des modes propres, on obtient le système 
suivant, dont les inconnues sont les coordonnées modales $q_{i}(t), \eta_{j}(t), i=1 \ldots N_{w}, j=1 \ldots N_{F}$ :

$$
\left\{\begin{array}{l}
\eta_{j}=-n_{0}^{j}-n_{P}^{j} V-\sum_{p, q=1}^{N_{w}} G_{p q}^{j} q_{p} q_{q} \\
\ddot{q}_{i}+2 \xi_{i} \omega_{s} \dot{q}_{i}+\omega_{i}^{2} q_{i}=-A_{0}^{i}-A_{P}^{i} V-\sum_{p=1}^{N_{F}} C_{p}^{i} \eta_{p} \\
+\sum_{p, q=1}^{N_{w}} D_{p q}^{i} q_{p} q_{q}+\sum_{p=1}^{N_{w}} \sum_{q=1}^{N_{F}} E_{p q}^{i} q_{p} \eta_{q}+Q_{i}
\end{array}\right.
$$

Un terme d'amortissement modal a été ajouté ( $\xi_{i}$ est le facteur d'amortissement du $i$-ième mode). Tous les coefficients qui apparaissent dans les équations précédentes $\left(n_{0}^{j}, n_{P}^{j}, G_{p q}^{j}, A_{0}^{i}, A_{P}^{i}, C_{p}^{i}, D_{p q}^{i}\right.$ et $\left.E_{p q}^{i}\right)$ sont des fonctions des $\left(\Phi_{i}, \Psi_{i}, \omega_{i}, \Upsilon_{j}, \zeta_{j}\right)$, connues analytiquement, non précisés ici par souci de concision. $Q_{i}$ correspond au forçage extérieur modal.

Les équations aux dérivées partielles initiales (5a, 5b) ont été remplacées par le problème discrétisé (10a, 10b) (équivalent si $N_{w}$ et $N_{F}$ sont infinis) constitués d'équations différentielles couplées par des termes nonlinéaires quadratiques. Ces termes, qui couplent les équations de flexion (10b) et les équations de membrane (10a) représentent le couplage flexion/membrane à la source des non-linéarités géométriques du problème. On peut éliminer les coordonnées de membrane $\eta_{j}$ entre les deux équations et obtenir un problème avec non-linéarités quadratiques et cubiques ne faisant intervenir que les $q_{i}$, formulation classique des équations de plaque et de coque de type von Kármán [6,7]. Ici, c'est la résolution avec la méthode asymptotique numérique, qui impose les nonlinéarités quadratiques, qui nous conduit à garder la formulation sous cette forme.

\section{Résolution}

Pour résoudre le problème et simuler à la fois le flambage et les vibrations non-linéaires, on décompose les variables en une partie statique $\left(\hat{q}_{i}, \hat{\eta}_{j}, \hat{V}\right)$, qui représente la solution du problème soumis à la précontrainte, et en une partie dynamique $\left(\tilde{q}_{i}, \tilde{\eta}_{j}, \tilde{V}\right)$, qui se rapporte aux oscillations autour de cette solution précontrainte. On écrit alors :

$q_{i}(t)=\hat{q}_{i}+\tilde{q}_{i}(t), \quad \eta_{j}(t)=\hat{\eta}_{j}+\tilde{\eta}_{j}(t), \quad V(t)=\hat{V}+\tilde{V}(t)$

La partie statique de la solution est obtenue en insérant les équations (11) dans (10a, 10b) et en annulant tous les termes dépendant du temps. On obtient alors un système d'équations non-linéaires algébriques qui permet de calculer les $\hat{q}_{i}$ et $\hat{\eta}_{j}$ et ainsi le déplacement statique $\hat{w}$ de la membrane à partir des équations (6), sous l'action des précontraintes introduites par les termes $n_{0}^{j}$ et $A_{0}^{i}$ (les précontraintes de fabrication), $n_{P}^{j} \hat{V}$ et $A_{P}^{i} \hat{V}$ (le chargement statique piézoélectrique). Si une solution $\hat{w}$ nonnulle est obtenue, alors il y a flambage. Ensuite, la partie dynamique du problème (les $\tilde{q}_{i}(t)$ et $\eta_{j}(t)$ ) est solution d'un système similaire aux équations (10), dont les coefficients dépendent des $\hat{q}_{i}$ et $\hat{\eta}_{j}$.

D'un point de vue pratique, la méthode de résolution suivante est suivie. La première étape consiste à calculer numériquement les coefficients $n_{0}^{j}, n_{P}^{j}, G_{p q}^{j}, A_{0}^{i}, A_{P}^{i}, C_{p}^{i}$, $D_{p q}^{i}$ et $E_{p q}^{i}$. Pour cela, les $\left(\Phi_{i}, \Psi_{i}, \omega_{i}, \Upsilon_{j}, \zeta_{j}\right)$ sont calculés à partir d'expressions semi-analytiques (à partir de fonctions de Bessel), en considérant la structure comme deux plaques annulaires connectés en $r=R_{1}$. Dans une seconde étape, le système statique est résolu par la Méthode Asymptotique Numérique (MAN) [8] implémentée dans le logiciel matlab [9]. Cela permet d'obtenir :

- la géométrie de la membrane précontrainte;

- les fréquences propres et les déformées modales de la structure précontrainte, en diagonalisant la partie linéaire du système dynamique.

La troisième étape est le calcul de la solution en régime forcé harmonique (les $\tilde{q}_{i}, \tilde{\eta}_{j}$ ). Pour cela, le forçage extérieur s'écrit soit $Q_{i}(t)=Q_{i m} \cos \Omega t$ (si c'est une force) ou $\tilde{V}(t)=V_{m} \cos \Omega t$ (si le système est excité par la couche piézoélectrique) et on cherche une solution périodique du système dynamique par équilibrage harmonique, sous la forme :

$$
\begin{aligned}
& \tilde{q}_{i}(t)=q_{i}^{(0)}+\sum_{h=1}^{H}\left(q_{i}^{c(h)} \cos h \Omega t+q_{i}^{s(h)} \sin h \Omega t\right) \\
& \tilde{\eta}_{j}(t)=\eta_{j}^{(0)}+\sum_{h=1}^{H}\left(\eta_{j}^{c(h)} \cos h \Omega t+\eta_{j}^{s(h)} \sin h \Omega t\right)
\end{aligned}
$$

où $H$ est le nombre d'harmoniques retenues. En introduisant les équations précédentes dans le système dynamique, on obtient un système algébrique non-linéaire d'inconnues $q_{i}^{(0)}, q_{i}^{c(h)}, q_{i}^{s(h)}, \eta_{j}^{(0)}, \eta_{j}^{c(h)}, \eta_{j}^{s(h)}, i=1 \ldots N_{w}$, $j=1 \ldots N_{F}, h=1 \ldots H$, que l'on résout avec la MAN [10]. Cela permet d'obtenir les courbes de résonance du système, présentées au paragraphe suivant.

\section{Premiers résultats : structure à stratification symétrique}

On s'intéresse à la structure représentée sur la figure 5 : une plaque en silicium et oxyde de silicium munie de deux éléments piézoélectriques similaires. La région centrale est composée de 11 couches et la zone annulaire de 3 couches. Ce système, bien que difficile à réaliser de manière pratique, est considéré ici en préliminaire à des études futures, car sa stratification est symétrique, ce 


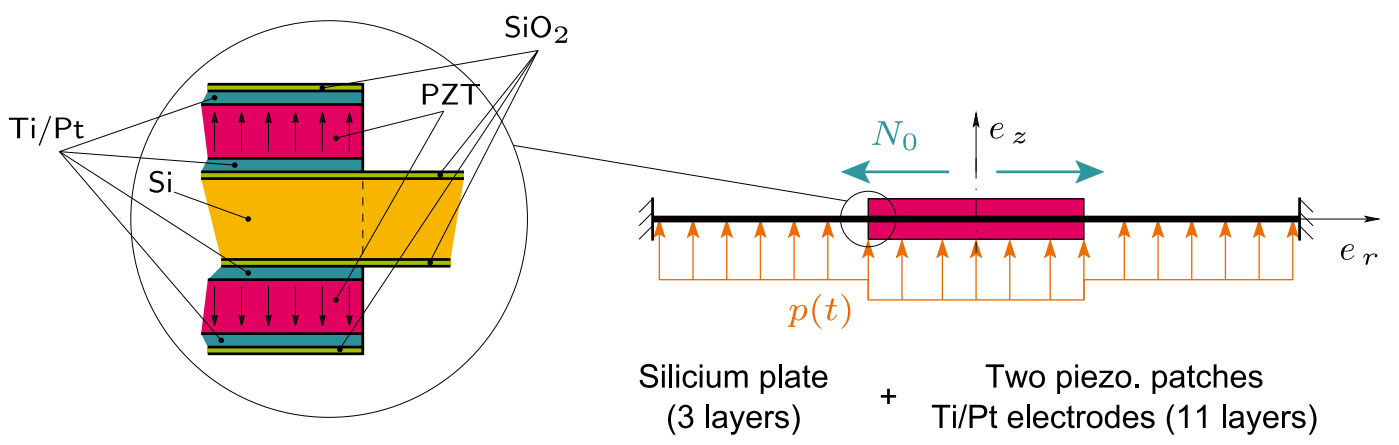

Fig. 5. Schéma du système.
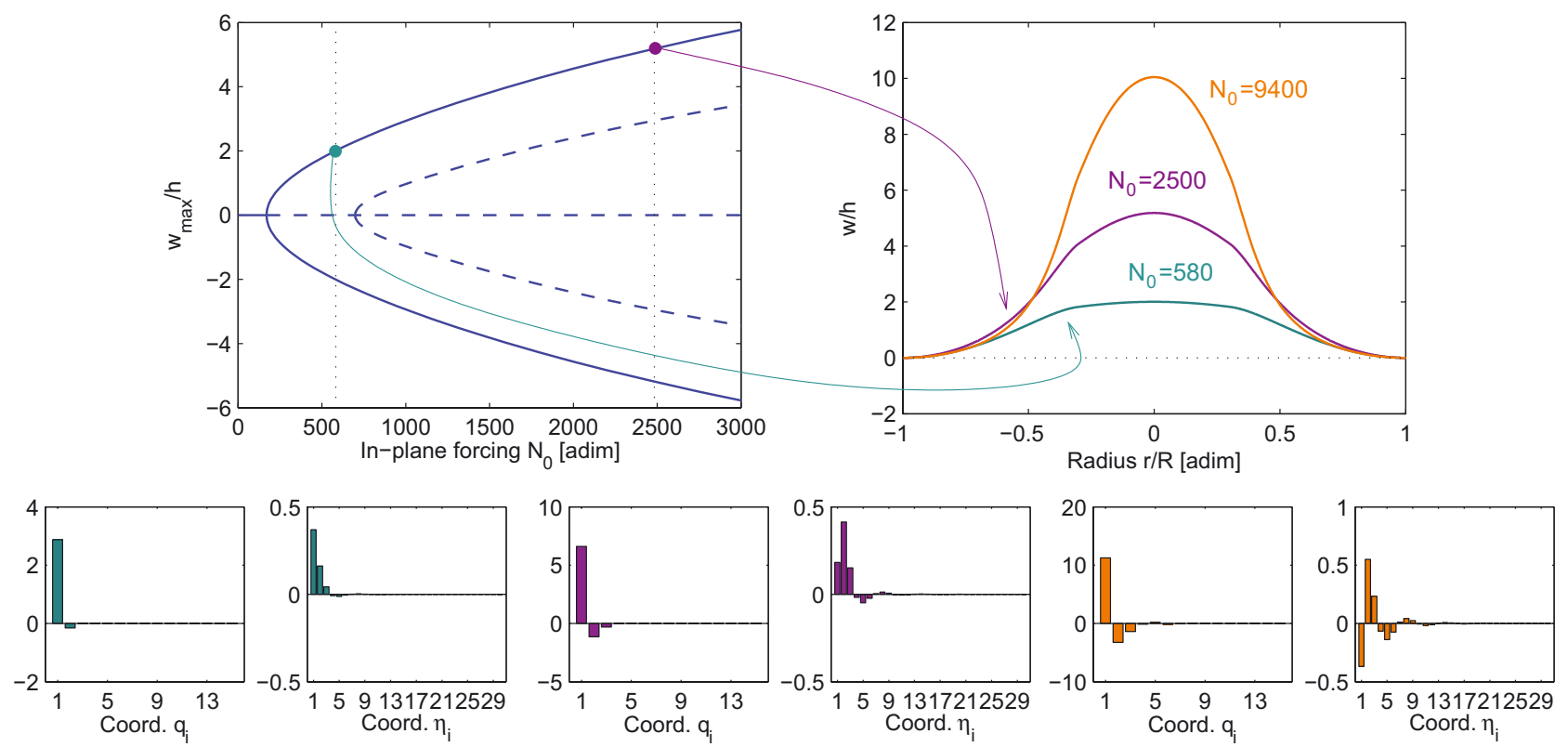

Fig. 6. Déplacement transverse du centre de la membrane en fonction de la précontrainte $N_{0}$. Déformées statiques en flambage, pour plusieurs précontraintes $N_{0}$; diagrammes de convergence (amplitude des $\hat{q}_{i}$ et $\hat{\eta}_{j}$ ).

qui permet de simplifier les calculs (la matrice de couplage flexion/membrane $\boldsymbol{B}$ est nulle, ce qui impose aussi $\left.C_{p}^{s}=D_{p q}^{s}=0\right)$. Le système est soumis à une précontrainte statique $N_{0}$, en imposant la même différence de potentiel aux deux couches PZT. Ensuite, un chargement sinusoïdal sous la forme d'une pression uniforme $p(t)=p_{0} \cos \Omega t$ est imposé.

La réponse statique de la membrane est représentée sur la figure 6 . On montre que pour une valeur critique de $N_{0}$ (autour de 200), on observe un flambage de la structure. Les profils obtenus pour plusieurs valeurs de $N_{0}$ sont aussi représentés, montrant l'influence non-négligeable de la raideur des pastilles (on observe une brusque variation de la pente du profil aux alentours de $r=0.3$, le rayon des pastilles).

La réponse dynamique, en terme de courbe de résonance, est représentée sur la figure 7 , pour plusieurs valeurs de $N_{0}$ et de $p_{0}$. On obtient des courbes incurvées vers les hautes fréquences (comportement raidissant) en dessous de la charge critique de flambage, lorsque la déformée statique est plane. On observe aussi que les fréquences propres diminuent lorsque $N_{0}$ augmente. Ensuite, lorsque la charge critique est dépassée (dans ce cas la première fréquence propre passe par zéro et se met à augmenter ensuite), le comportement non-linéaire devient assouplissant (courbe incurvée vers les basses fréquences). Cela est un résultat classique pour les structures nonplanes comme les coques [11].

Enfin, la figure 7 montre aussi les oscillations du centre de la membrane au cours du temps, à la résonance, pour une valeur de $N_{0}$ provoquant le flambage. On observe une certaine dissymétrie, qui se retrouve sur l'évolution de profil de la membrane au cours d'une oscillation. Le diagramme de convergence montre que $H=5$ harmoniques et $N_{w}=3$ modes sont suffisants pour simuler correctement le comportement du système. 

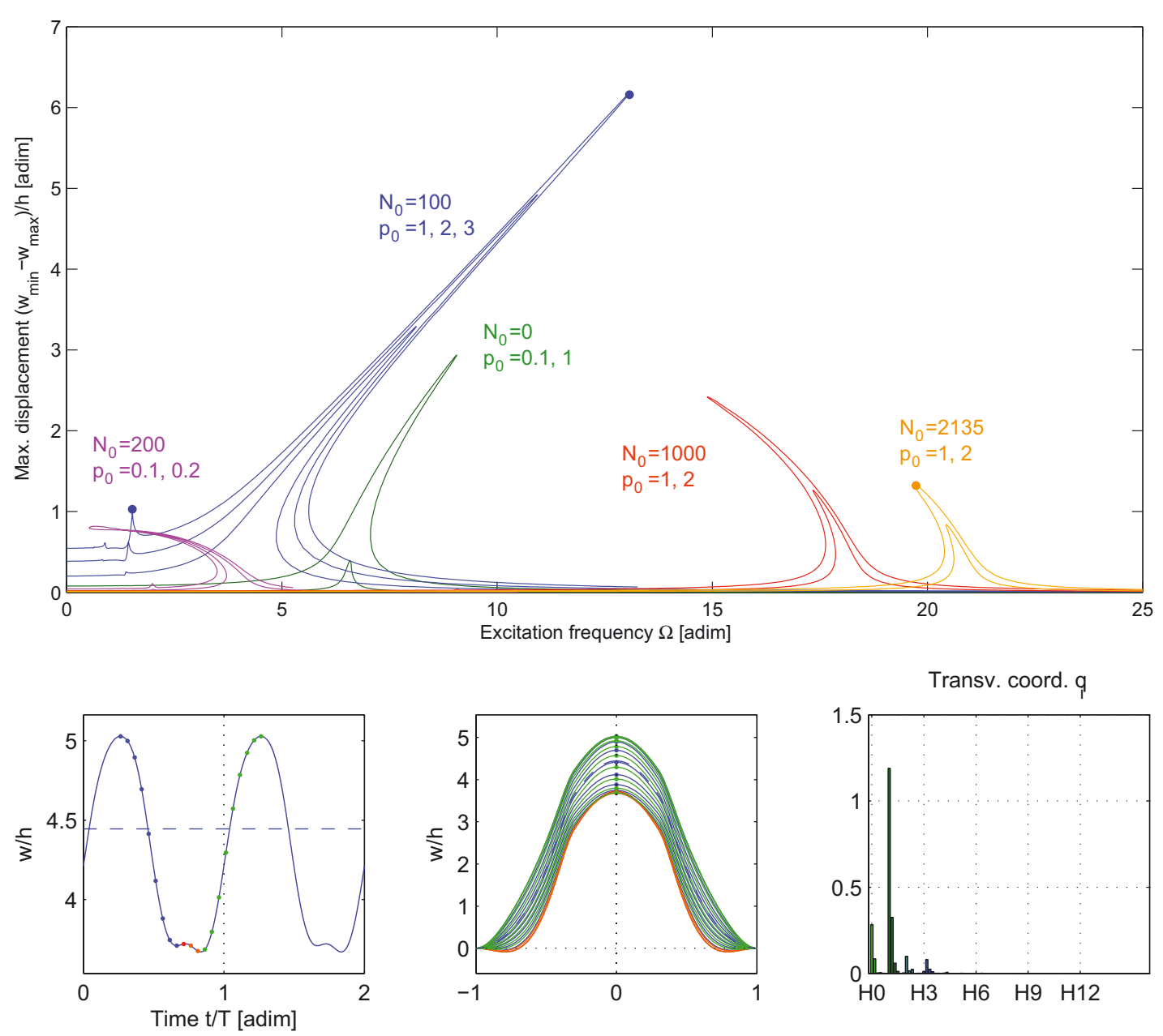

Fig. 7. Réponse en fréquence autour de la première résonance, pour plusieurs précontraintes $N_{0}$ et plusieurs amplitudes $p_{0}$ du forçage. Oscillations à la résonance, autour de la position statique flambée, pour $N_{0}=2135$; profil de la déformée à différents instants; convergence du calcul : amplitude des $q_{i}^{(0)}, q_{i}^{c(h)}, q_{i}^{s(h)}$.

\section{Références}

[1] L. Nicu, C. Bergaud, Modeling of a tuning fork biosensor based on the excitation of one particular resonance mode, J. Micromech. Microeng. 14 (2004) 1-10

[2] C. Ayela, L. Nicu, Micromachined piezoelectric membranes with high nominal quality factors in newtonian liquid media: a lamb's model validation at the microscale, Sens. Act. B Chem. 123 (2006) 860-868

[3] L. Nicu, M. Guirardel, F. Chambosse, P. Rougerie, S. Hinh, E. Trevisiol, J.-M. François, J.-P. Majoral, A.-M. Caminade, E. Cattan, C. Bergaud, Resonating piezoelectric membranes for microelectromechanically-based bioassay: detection of streptavidin-gold nanoparticles interaction with biotinylated dna, Sens. Act. B Chem. 110 (2005) 125-136

[4] J.N. Reddy, Mechanics of laminated composite plates, CRC press, 1997

[5] C.Y. Chia, Nonlinear analysis of plates, Mc Graw Hill, 1980
[6] O. Thomas, S. Bilbao, Geometrically non-linear flexural vibrations of plates: in-plane boundary conditions and some symmetry properties, J. Sound Vib. 315 (2008) 569590

[7] C. Camier, C. Touzé, O. Thomas, Non-linear vibrations of imperfect free-edge circular plates and shells, Eur. J. Mech. A/Solids 28 (2009) 500-515

[8] B. Cochelin, N. Damil, M. Potier-Ferry, Méthode asymptotique numérique, Hermès, 2007, In French

[9] R. Arquier, Manlab : logiciel de continuation interactif (manuel utilisateur), Technical report, Laboratoire de Mécanique et d'Acoustique, CNRS, http://www. Ima. cnrs-mrs.fr/ manlab, 2005, In French

[10] B. Cochelin, C. Vergez, A high order purely frequential harmonic balance formulation, J. Sound Vib., 2009, In press

[11] C. Touzé, O. Thomas, Non-linear behaviour of freeedge shallow spherical shells: effect of the geometry, International Journal of non-linear Mechanics 41 (2006) 678-692 